\title{
What Scientists Want From Their Research Ethics Committee
}

Article in Journal of Empirical Research on Human Research Ethics · April 2006

DOI: 10.1525/jer.2006.1.1.67 · Source: PubMed

CITATIONS

44

2 authors:

\section{Patricia Keith-Spiegel}

50 PUBLICATIONS 1,354 CITATIONS

SEE PROFILE
READS

216

Some of the authors of this publication are also working on these related projects: 


\section{What Scientists Want from Their Research Ethics Committee}

\author{
Patricia Keith-Spiegel and Gerald P. Koocher \\ Children's Hospital, Boston \\ BARBARA TABACHNICK \\ California State University, Northridge
}

\begin{abstract}
WHEREAS INVESTIgATORS HAVE DIRECTED considerable criticism against Institutional Review Boards (IRBs), the desirable characteristics of IRBs have not previously been empirically determined. A sample of 886 experienced biomedical and social and behavioral scientists rated 45 descriptors of IRB actions and functions as to their importance. Predictions derived from organizational justice research findings in other work settings were generally borne out. Investigators place high value on the fairness and respectful consideration of their IRBs. Expected differences between biomedical and social behavioral researchers and other variables were unfounded. Recommendations are offered for educating IRBs to accord researchers greater respect and fair treatment.
\end{abstract}

KEY WORDS: ethics committee, IRB, fairness, organizational justice

Received November 20, 2005, revised January 16, 2006

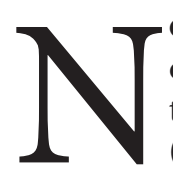

OT ALL SCIENTISTS ARE DISPLEASED with the operating procedures and competence of their own Institutional Review Board or IRB. (Research Ethics Committees are known by various names. We use the term IRB throughout this article.) Characteristics of an exemplary IRB, however, have not been systematically studied. On the other hand, it is easy to find critics of IRBs, and complaints about IRBs regularly appear in published scientific commentaries.

Specific criticisms of IRB members and administrators have included shallow and rushed reviews of submitted protocols, favoring the protocols of selected colleagues, concealing conflicts of interest, making unreasonable requests for changes, imposing excessive bureaucratic requirements, and incompetence (DuBois \& James, 2004;
Ferraro, Szigeti, Dawes \& Pan, 1999; Hayes, Hayes, \& Dykstra, 1995; Kancelbaum, 2002; Malouff \& Schutte, 2005; OHRP, September, 2000a; Palca, 1996; Silberner, 1998). Cohen (1998) cites specific concerns expressed at a summit meeting titled "IRBs: All checks and no balances." These include censorship of research topics, inconsistent decision-making, harassment, lack of accountability, biases, ineffective communication with investigators, lack of knowledge about research, unrealistic assessment of risks, lack of appeal mechanisms, wide variations in the interpretation of the federal regulations, and a focus on participants' rights to the neglect of scientific merit. Other criticisms include excessive protocol dwell time, rudeness, using "local standards" as a justification for idiosyncratic rulings, and being overly conservative and protective of the institution and research participants (Edwards, Kirchin, \& Huxtable, 2004; Keith-Spiegel \& Koocher, 2005; Wainwright \& Saunders, 2004).

Expressions of concern about the ability of IRBs to adequately perform their considerable duties are hardly of recent origin. Not long after the National Research Act of 1974 mandated the establishment of IRBs in research institutions receiving federal research funds, Gray, Cooke, and Tannenbaum (1978) surveyed the IRB process at 61 institutions. They reported that those IRBs making more decisions requiring changes and modifications to protocols also received more negative ratings by investigators. However, rather than linking IRB shortcomings to justified dissatisfaction on the part of investigators, they presumed that negative attributes occurred, "when investigators do not see a link between IRB actions and the protection of subjects" (p. 1099).

Most complaints expressed by investigators deal with IRB procedures and interpersonal interactions perceived by investigators as unfair. The organizational justice literature is useful for understanding complaints about IRBs, as well as conceptualizing an "ideal IRB." We propose that if IRB members had a full awareness of the influence of injustice issues on how investigators perceive them, and became more attentive to what investigators find most essential to proper IRB functioning, criticisms of IRBs would diminish (KeithSpiegel \& Koocher, 2005). However, one must first determine if justice issues are, in fact, as important to

Journal of Empirical Research on Human Research Ethics, PP. 67-82. PRINT ISSN 1556-2646, ONLINE ISSN 1556-2654. (C) 2006 BY JOAN SIEBER. ALL RIGHTS RESERVED. PLEASE DIRECT ALL REQUESTS FOR PERMISSIONS TO PHOTOCOPY OR REPRODUCE ARTICLE CONTENT THROUGH THE UNIVERSITY OF CALIFORNIA PRESS'S RIGHTS AND PERMISSIONS WEBSITE, WWW.UCPRESS.EDU/JOURNALS/RIGHTS.HTM 
most scientists as they are to employees functioning in very different work settings.

\section{Theoretical Foundations}

\section{Procedural Justice}

Perceptions of procedural justice arise from a belief that decisions flow from clear policies, applied evenly, without bias or prejudice (Tyler \& Bies, 1989; Tyler \& Lind, 1992). People do not usually direct hostility against those who do what they were supposed to do (Tyler \& Bies, 1989; Tyler \& Lind, 1992). Nor do people usually hold others responsible for failing to do something when it was not possible to do it (Folger \& Cropanzano, 1998). However, even the most carefully developed and detailed policies cannot cover every possible circumstance that might arise. Hence, the scrupulous application of procedural justice can reduce negative reactions to adverse decisions. Levanthal $(1976,1980)$ generated a list of process characteristics that foster the perception of procedural fairness. These include: consistency, lack of bias, accuracy, procedures for correcting errors, representativness, and adherence to basic ethical standards. This list appears to describe how investigators might characterize a fair IRB.

One of the significant properties of procedural justice involves what Thibaut and Walker (1975) referred to as voice. People are more willing to accept a negative outcome when they feel that they had an opportunity to present their case and be heard (Bies, 1987). The willingness and ability of decision makers, such as IRBs, to listen and seriously consider what the person has to say becomes a critical factor. It reduces loss of self-esteem caused by a negative outcome because the individual's views were at least implicitly acknowledged (Folger, et al., 1998). Although IRBs should offer investigators an adequate voice, a common complaint focuses on limits to such opportunities.

\section{Interactional Justice}

Another type of perceived unfair treatment, interactional injustice, involves the social component of procedural justice (Folger, et al., 1998; Greenberg, 1996; Tyler, et al., 1989; Tyler, Rasinski, \& Spodick, 1985 ). Interactional justice involves the behavioral aspects of the decision process. Was the person who received a negative decision treated in a sensitive and dignified manner? Or did the person receive dismissive, arrogant treatment with little respect or empathy? Any evidence of caring will alter the affected person's perceptions of the decision-makers, even when the outcome is negative (Lind \& Tyler, 1988).

Folger, et al. (1998) divide interactional justice into sub-categories. Interpersonal sensitivity refers to the degree of politeness and respectfulness people experience. When people feel dealt with offhandedly or disrespectfully, they become prone to poor attitudes and poor performance. Only a modest degree of empathy can have significant effects in perceived interactional justice. Interpersonal sensitivity conveys a strong symbolic message that can have significant meaning to a person, even one who has been the recipient of a negative outcome.

Justification is the second component of interactional injustice. It involves offering the recipient an explanation of the rationale for making a particular decision. Those with the power to make decisions may feel that they do not need to offer any explanations because they have final authority, not subject to question. However, people who receive a sincere and adequate reason for a negative outcome more readily accept it (Bies, Shapiro, \& Cummings, 1988). Inadequate explanations make the recipient feel marginalized and produce hostile feelings towards the source of the unsatisfying outcome (Tyler, et al., 1992). Without a full explanation people will attribute their own motives and intentions to decisionmakers, and these are usually distinctly negative, perhaps to the point of disregarding the decision. For example, if the IRB does not approve a protocol and offers curt, perfunctory reasons why the proposed procedure was unacceptable, the investigator may start plotting ways to get around the IRB, such as submitting a new proposal with an "acceptable" consent procedure, but using the original one in the actual study. However, suppose the IRB decides to postpone approval of a protocol while awaiting feedback from an outside expert. If the IRB communicates this decision to the investigator, the IRB may avoid attributions of slowness, incompetence, lack of uncaring, or insensitivity. An even stronger positive message would involve the inclusion of an apology for the delay (interpersonal sensitivity). Additional efforts to attend to matters of interactional justice may yield high returns in the form of an enhanced positive reputation of the IRB which, in turn, contributes to improved IRB/investigator relationships and, perhaps, compliance.

\section{Study Setting and Population Differences}

Most organizational justice field research has been conducted in factories, business settings, or service agencies characterized by a clear line of command and constant supervision of employees. However, the typical research 
settings in which IRBs function are institutions of higher education, including research hospitals associated with universities. Such facilities do not typically closely supervise or externally monitor investigators' work. Once an IRB has completed its evaluation and reached a final decision it typically has little further direct involvement in research programs except for required annual monitoring, which is often perfunctory. Can findings from typical venues of organizational research be generalized to academic settings?

The people most often studied in organizational justice research occupy subordinate positions subject to evaluation or otherwise leveraged by others holding some form of power to make judgments or disperse resources. Research scientists, however, do not fit neatly into this pattern. IRBs can include colleagues who are junior in position and status to the investigator whose proposals they must evaluate. IRBs also include lay members of the public and professionals from other fields. IRBs, then, may be perceived as unjust because investigators do not necessarily hold their members in high esteem. Investigators may more easily become upset when negative outcomes flow from those to whom they feel superior in status, specific content knowledge, or authority. This is not to say that IRBs are without considerable power over investigators. IRBs have the authority to expedite, delay, suspend, or discontinue altogether an investigator's work.

Because IRBs hold such important gatekeeping power, and because investigators are so dependent on research productivity to define their professional identities, IRBs may become especially vulnerable to perceived injustice because, for investigators, the stakes are high. When an IRB makes a negative decision, investigators can easily perceive the action as disrespectful or critical of their work. Because of people's reluctance to attribute negative outcomes to their own shortcomings (e.g., Weiner, 1995), IRBs may be particularly vulnerable to perceptions that they acted unfairly.

IRBs function under unusual pressures that could strongly affect investigators' perceptions of the fairness of IRB decisions. They operate largely under policy set at the federal level. The demands placed on IRBs are considerable and increasing (see, for example, Compliance oversight procedures issued by OHRP, 2000b). IRBs review and judge each protocol on dimensions of risk, informed consent procedure, equity, privacy and confidentiality, vulnerability of subjects, and appropriateness of incentives. IRB members and the administrators who staff them remain well aware that doing an inadequate job could result in suspension or withdrawal of their Assurance of Compliance. Investigators could face suspension or loss of their privilege of conducting research. Because IRBs cannot constantly oversee what investigators actually do behind laboratory doors or in the field, some IRBs may seek to avoid the risk of criticism or bad publicity by restricting or disapproving protocols that have some controversial feature, even those that are likely to be in compliance with federal standards, taking advantage of the provision that allows for decisions based on "local context" or "community standards."

\section{A Search for the Ideal IRB: Method and Hypotheses}

A list of 45 characterizations of IRB functions and activities was generated from the literature and from the IRB documents of a number of institutions, and reviewed by volunteer colleagues. This list formed the basis of a questionnaire (see Table 1). The items were clustered into eight a priori themes, and the questionnaire was designed to assess the relative importance to the investigators of these themes or factors. Proposed factors were: procedural justice (how the decision-making process is carried out); absence of bias (a feature of procedural justice); pro-science sensitivity and commitment; interactional justice (interpersonal sensitivity and justification); formalities (committee's formal functioning, structure, and composition); upholding of rights of human research participants; IRB outreach (offering services beyond those mandated); and competence (how competently the IRB performs its functions).

Because all of the items might be regarded by most investigators as important, the directions to the respondents were designed to maximize response variability. The directions acknowledge that most of the items are likely to be seen as important. Respondents are then asked to spread their ratings across the full range of response categories.

Questionnaires, along with a cover letter and franked return envelope, were sent to a large, national sample of biomedical and social behavioral scientists. One thousand two hundred and eighty three randomly selected principal investigators (PIs) of currently funded PHS research and 1,000 randomly selected members of the American Psychological Society were sent a survey asking them to rate the importance in their own work of each item on a scale of 1 ("not important") to 7 ("extremely important"). ${ }^{1}$ Respondents were also asked to rate how their own IRB compared to their ideal IRB, and to identify their research specialty type (e.g., educational, biomedical, social behavioral), IRB service over the past five years, number of years conducting research in a professional capacity, percentage of exempt research 
TABLE 1. Item means and standard deviations ranked within apriori factors. ${ }^{3}$

\section{Procedural Justice}

1. An IRB that reviews protocols in a timely fashion $(6.43,0.80)$

6. An IRB that conducts a conscientious and complete review of protocols $(5.86,1.24)$

9. An IRB that gives a complete rationale for any required changes to or disapprovals of protocols $(5.73,1.21)$

12. An IRB that includes a complete rationale when it denies or mandates changes in a protocol based on criteria that are more stringent than or different from federal research policy (i.e., application of "local standards") $(5.59,1.35)$

16. An IRB that is open to reversing its earlier decisions (i.e., willing to consider investigator appeals) $(5.52,1.34)$

17. An IRB that invites investigators to present their position whenever a question or concern about a research protocol arises $(5.51,1.33)$

27. An IRB that recognizes when it lacks sufficient expertise to evaluate a protocol and seeks outside experts $(5.28,1.41)$

Interpersonal Justice

8. An IRB that responds in a timely manner to investigators' inquiries about its processes and decisions $(5.80,1.15)$

10. An IRB that works with investigators to find mutually satisfying solutions whenever disagreements exist $(5.71,1.27)$

20. An IRB that treats investigators with respect $(5.45,1.45)$

25. An IRB that acknowledges full responsibility for its errors or delays in processing protocols and attempts to correct them as expeditiously as possible $(5.33,1.44)$

35. An IRB that is open and pleasant in its interactions with investigators $(4.72,1.61)$

Bias

2. An IRB with members who do not allow personal biases to affect their evaluation of protocols $(6.17,1.10)$

21. An IRB whose members hold no preconceived biases against particular research topics $(5.45,1.46)$

22. An IRB that requires members to abstain from evaluating protocols whenever a real or apparent conflict-of-interest arises $(5.44,1.46)$

23. An IRB whose members hold no preconceived biases against particular research techniques $(5.43,1.47)$

28. An IRB that is open to innovative approaches to conducting research $(5.28,1.43)$

Pro-Science Sensitivity

3. An IRB that does a good job of upholding participants' rights while, at the same time, facilitating the conduct of research $(6.10,1.11)$

4. An IRB that does not use its power to suppress research that is otherwise methodologically sound and in compliance with federal policy whenever it perceives potential criticism from outside the scientific community $(6.08,1.19)$

13. An IRB that views itself as an investigator's ally rather than as a hurdle to clear $(5.57,1.44)$

33. An IRB that shows considerable evidence that the advancement of science is part of its mission $(4.82,1.79)$

37. An IRB that shows empathy with the difficulties that can present themselves during the design and conduct of research $(4.66,1.60)$

IRB Competence

5. An IRB with members who are very knowledgeable about IRB procedures and federal policy $(6.01,1.16)$

14. An IRB that conducts a conscientious analysis of potential benefits weighed against potential risks before making decisions (5.54, 1.29)

19. An IRB that can competently distinguish exempt from nonexempt research $(5.48,1.44)$

31. An IRB that ensures that at least one member is knowledgeable about the content domain of submitted protocols $(5.13,1.55)$

32. An IRB whose members arrive at meetings well-prepared $(5.07,1.52)$

36. An IRB with a Research Compliance Officer (or staff member in charge of IRB functions) who has a research background $(4.68,1.66)$

38. An IRB that is composed primarily of members regarded as highly competent investigators $(4.46,1.70)$

40. An IRB that provides a comprehensive training program for its new members $(4.34,1.64)$

IRB Outreach

26. An IRB that offers information to improve the chances of gaining IRB approval $(5.31,1.45)$

41. An IRB that offers consultation during the development of research protocols and grant applications $(4.30,1.76)$

43. An IRB that offers investigators opportunities to be educated about federal research policy $(4.03,1.68)$

44. An IRB that offers editorial suggestions regarding consent documents and protocols (typos, grammar, clarity, etc.) (3.20, 1.82)

IRB Formal Functioning, Structure, and Composition

11. An IRB whose members fully understand and act within the scope of their function $(5.67,1.27)$

18. An IRB that maintains accurate records $(5.50,1.50)$

24. An IRB that is allocated sufficient resources to carry out its functions $(5.38,1.44)$

34. An IRB that requires that its Chair be an experienced investigator $(4.75,1.76)$

39. An IRB that monitors the progress of each approved research project in line with federal policy $(4.39,2.16)$

42. An IRB that has a diverse membership (i.e., includes women, minorities and junior and senior members of the institution) $(4.07,1.93)$

45. An IRB that is composed of more than one public member $(2.68,1.69)$

Upholding the Rights of Human Participants

7. An IRB that views protection of human participants as its primary function $(5.80,2.83)$

15. An IRB that takes timely and appropriate action whenever scientific misconduct is alleged $(5.52,1.42)$

29. An IRB that applies appropriately flexible standards regarding voluntary and informed consent requirements (e.g., required wording is not as demanding for minimal risk research as it is for more risky research) $(5.23,1.52)$

30. An IRB that takes timely action when an investigator has violated its decisions $(5.22,1.51)$ 
conducted, and gender. Of the 910 returned surveys returned, 886 were usable for analysis purposes, for a return rate of $38.8 \%$.

The useable sample included 493 men (55.6\%), 375 women (42.3\%), and 18 respondents (2\%) who declined to record their sex. There were 565 social/behavioral scientists $(63.8 \%$ of the sample), 261 biomedical researchers $(29.5 \%), 11$ educational researchers $(1.2 \%)$ and 26 who reported "other" (2.9\%). Twenty-three researchers $(2.6 \%)$ failed to indicate research area. The greatest number of respondents came from research universities $(45.6 \%)$, followed by comprehensive universities (20.3\%) and 4-year colleges (12.8\%). In all $78.7 \%$ reported working at a college or university. Eleven percent report working in a hospital or medical facility, $2 \%$ for a private company, and $5 \%$ on other settings, such as a community agency.

Most respondents (66.3\%) reported they had not served on an IRB in the past 5 years, however $73(8.2 \%)$ failed to respond to this item. Fifty-eight (6.5\%) had served for one year, $52(5.8 \%)$ had served for two years, $38(4.3 \%)$ reported serving three years, $26(2.9 \%)$ reported serving 4 years, and 51 (5.8\%) reported serving five years. One respondent claimed 12 years of service. Exempt research was not the norm; only one-quarter of the researchers spent more than half of their research time in exempt research.

\section{Expected Sample and Other Differences}

Despite the apparent differences between our sample of scientific investigators and the typical samples tapped in most organizational justice research, our primary hypothesis was that a just and fair IRB is very important to all those who conduct research. However, we also expected to find at least some differences among types of investigators (i.e., biomedical or BM v. socialbehavioral or SB) and research settings (e.g., research university v. comprehensive university).

SB scientists often complain that IRBs hold them to unnecessarily strict standards, not taking into account that potential risks in most SB research are virtually nonexistent. Thus, we predicted that BM researchers would give greater importance than SB scientists to formalities, primacy of according rights to human subjects, IRB outreach efforts, and competence. We also predicted that $\mathrm{BM}$ investigators would be more concerned with issues related to risk management due to the higher level of risk inherent in many BM studies, relative to $\mathrm{SB}$ research.

Because IRBs at research universities may have better organization and staffing and possibly more than one
IRB, permitting more efficient division of labor, we hypothesized that investigators at research universities would rate their own IRBs higher than would investigators from other settings, such as comprehensive universities and 4-year colleges where resources are often in short supply, leaving IRBs understaffed and possibly less well-trained. We also expected to find that the ratings of investigators' own IRB (relative to the ideal) would be higher for BM than SB investigators, especially within research universities where SB investigators have complained about being judged by inappropriately strict standards (Cohen, 1998).

Because so many IRB complaints seem fairness-related, we hypothesized that investigators who expressed dissatisfaction with their IRBs would show even more concern with IRB justice issues than would those who expressed more satisfaction. We hypothesized that those who are used to full protocol reviews would complain less because they are more used to being scrutinized, whereas those whose work falls into the exempt category may complain when their work does not gain swift approval. We also expected to find that those who did more research falling into the exempt category would have more concern about bias and competence issues.

We expected that those with no IRB experience would express more concern about procedural justice, bias, interactional justice, and competence than those with IRB experience because they may have less understanding of the IRB system and procedures. We also expected that those who had served on IRBs within the last 5 years would rate more highly the items dealing with ensuring the rights of human participants and the formalities associated with running an IRB. We did not expect to find gender differences.

\section{Results}

The analysis strategy began with confirmation (via a confirmatory factor analysis conducted through structural equation modeling) of the eight-factor model to account for shared variance among the 45 items of the questionnaire about the importance of IRB characteristics. Once an acceptable model was achieved, factor scores were computed and adjusted to match the original scale of the items. Hypothesized differences among factors and types of researchers were examined through MANOVA. Factor scores also were used as multivariate predictors of hypothesized group differences in discriminant analyses. This analysis provides information about all predictors adjusted for one another, which is helpful when predictors are correlated. Type I error rate was controlled by setting $\alpha=.05$ for the multivariate 
test of each planned comparison and $\alpha=.05$ divided by number of predictors for univariate tests of predictors. Chi-square and logistic regression analyses were used to evaluate planned comparisons regarding relationships among groups.

Table 1 shows means and standard deviations for the 45 questions regarding importance of characteristics of IRBs sorted into the a priori factor categories. Codes of 1 to 7 represent ratings of increasing importance of the described characteristic. Most items had strong negative skewness; that is most items were considered at least fairly important by most researchers.

\section{Factor Structure of the Questionnaire}

An acceptable model was generated when the eight proposed factors were permitted to correlate. One item was omitted from the model: "application of appropriately flexible standards" was expected to load on the "competence" factor, but did not. Although attempts to improve the model through a cross-validation strategy resulted in a better model fit, that improvement was offset by loss in interpretability. Therefore, the minimally altered model was used for subsequent analyses. The root mean square error of approximation for the model was .059 with a $90 \%$ confidence interval of .057 to .061 . Values less than .10 for a sample of this size are considered acceptable. ${ }^{2}$

Figure 1 shows the standardized regression coefficients for the model. Table 2 shows correlations among factors. Note that some of the factors are quite highly correlated; competence shares a great deal of variance with both procedural justice and formalities (committee's formal functioning, structure, and composition).

\section{Importance of Justice Issues}

A two-way within-between-subjects MANOVA was performed on adjusted factor scores. The three-level within-subjects independent variable (IV) was type of factor: justice vs. competence vs. other factors. For the "justice" level, adjusted factor scores were averaged over four factors: procedural justice, absence of bias, pro-science sensitivity, and interactional justice. The "other" level was comprised of the average adjusted factor scores for formalities, IRB outreach, and sensitivity to right of human participants. Competence was the adjusted score for that single factor. The between-subjects IV was type of researcher: social/behavioral vs. biomedical.

There was a strong main effect of type of factor, multivariate $F(2,869)=560.08, p<.001$, partial $\eta^{2}=.56$, with a $95 \%$ confidence interval (CI) around partial $\eta^{2}$ from .52 to .60 . As predicted, justice issues were rated to be more important $(M=5.53, S E=0.03)$ than were competence $(M=4.81, S E=0.03)$ or other issues $(M=$ $4.72, S E=0.03)$. There also was a statistically significant interaction between justice issues and type of researcher, multivariate $F(2,869)=13.44, p<.001$, partial $\eta^{2}=.03$ with a $95 \%$ CI from .01 to .05 . As seen in Figure 2, the difference in importance of justice-related vs. other issues was greater on average for the 601 social/behavioral researchers than for the 271 biomedical researchers. A small but statistically significant main effect of type of researcher also was noted, $F(1$, $870)=8.84, p=.003$, partial $\eta^{2}=.01$ with a $95 \% \mathrm{CI}$ from 0 to .03 . On average, biomedical researchers assigned greater importance to items on the questionnaire $(M=$ $5.10, S E=0.05)$ then $\mathrm{did}$ social/behavioral researchers $(M=4.94, S E=0.03)$.

The four factors comprising justice were analyzed in a one-way within-subjects MANOVA, followed by post-hoc comparisons among all four factors using a Scheffé adjustment. Overall, there were statistically significant differences in rated importance of the four justice factors, multivariate $F(3,883)=89.54, p<.001$, partial $\eta^{2}=.23$ with a $95 \%$ CI from .19 to .28 . All Scheffé-adjusted contrasts were statistically significant $(p<.001)$ except between the procedural and interactional justice factors. Table 3 shows mean differences and partial $\eta^{2}$ with its associated 95\% CI for the six comparisons. Procedural and interactional justice were given the highest importance $(M=5.66, S E=.03)$, followed by pro-science sensitivity $(M=5.54, S E=.03)$ and absence of bias $(M=5.27, S E=.03)$.

\section{Differences Among Groups}

Satisfied vs. Dissatisfied Researchers. We hypothesized that the group of 92 dissatisfied researchers who rated their own IRBs in the two least favorable categories would place higher importance on items in three factors (procedural justice, interactional justice, and absence of bias) than would the group of 324 researchers who rated their IRBs in the two most favorable categories. A small but statistically significant multivariate difference between groups was found, multivariate $F(3,412)=$ 3.44, $p=.017, \eta^{2}=.02$ with $95 \%$ CI from 0 to .06 . However, only one of the hypothesized factors, interactional justice, would significantly degrade discrimination between groups if absent, $F$-to-remove $(1,412)=8.51$, $p<.017, \eta^{2}=.02$ with $95 \%$ CI from 0 to .05 , indicating only a small association between group membership and rating of importance of interactional justice. As predicted, dissatisfied researchers placed greater importance on items comprising interactional justice 
$(M=5.55, S D=0.81)$ than did satisfied researchers $(M=5.22, S D=0.97)$.

Percentage of Exempt Research. Two separate groupings of researchers were evaluated with respect to differences associated with percentage of exempt research, with differences hypothesized on two factors: absence of bias and competence. The first grouping contrasted the 268 researchers who conducted no exempt research with the 559 who conducted some. No statistically significant multivariate difference was found between the two groups, $p=.93, \eta^{2}=0$. The second grouping contrasted the group of 508 researchers whose exempt research consisted of $25 \%$ or less of their research with the group of 319 researchers whose exempt research consisted of more than $25 \%$ of their research. Again, no significant group difference was found, $p=.47, \eta^{2}=0$. Because significant differences between groups were not observed, there was no test of the prediction that differences would be larger for research universities and hospitals than for comprehensive universities and four-year colleges.

IRB Experience. Differences on several factors were hypothesized between the group of 587 researchers who had not served on an IRB in the past five years and the group of 168 who had served more than one year. We expected that those with no IRB experience would give higher ratings to factors representing procedural justice, absence of bias, interactional justice, and competence than those with IRB experience. We also expected that those with IRB experience would give higher ratings to factors representing formalities and primacy of rights of human participants. We found no statistically significant group difference when tested multivariately, $p=.51, \eta^{2}=0$.

Social/Behavioral Scientists vs. Biomedical Researchers. We predicted that biomedical researchers would assign greater importance than social scientists to four factors: formalities, primacy of rights of human subjects, IRB outreach, and competence. We found a small but statistically significant difference between the 601 social scientists and the 271 biomedical researchers, multivariate $F(4,867)=$ $6.85, p<.001, \eta^{2}=.03$ with $95 \%$ CI from .01 to .05 . The only unique source of difference was the factor with items representing formalities of the IRB, F-to-remove $(1,867)=$ $12.33, p<.0125, \eta^{2}=.01$ with $95 \%$ CI from 0 to .03 . As predicted, biomedical researchers gave higher ratings to formalities $(M=4.53, S D=1.07)$ than did social/behavioral scientists $(M=4.24, S D=1.07)$. No significant prediction of group membership was provided by the remaining three hypothesized factors, $p>.0125$.
We also hypothesized differences between $\mathrm{BM}$ and $\mathrm{SB}$ respondents on a set of items representing "risk management." These issues were not particularly associated with the factors, but would represent IRB characteristics that minimize the institution's research programs exposure to adverse action by federal agencies. Higher ratings were predicted for biomedical researchers as compared to $\mathrm{SB}$ researchers on items dealing with the provision of a comprehensive training program for IRB members, the maintenance of accurate records, taking timely action when violations occur, assigning protection of human participants as the primary IRB function, sufficient allocation of resources for IRBs, the availability of consultation services during the preparation of grant applications and research protocols, and monitoring of projects in compliance with federal policy. Lower ratings by $\mathrm{BM}$ researchers as compared to $\mathrm{SB}$ researchers were predicted for items dealing with more flexible standards for voluntary and informed consent and risk assessment, inclusion of public members, competent differentiation between exempt and nonexempt research, and resisting the suppression research for fear of outside criticism. A statistically significant multivariate difference was found between groups for the set of 12 items, multivariate $F(12$, $859)=4.83, p<.001, \eta^{2}=.06$, with $95 \%$ CI from .02 to .08 . However, only two items uniquely discriminated the groups. Biomedical researchers indeed showed more concern $(M=5.69, S D=1.32)$ than social/behavioral scientists $(M=5.23, S D=1.47)$ with allocation of sufficient resources, F-to-remove $(1,864)=10.95, p<.005, \eta^{2}=.01$, with $95 \%$ CI from 0 to .03 . Social/behavioral scientists showed more concern $(\mathrm{M}=5.54, \mathrm{SD}=1.41)$ than biomedical researchers $(M=5.31, S D=1.46)$ with the competence of making the distinction between exempt and nonexempt research, F-to-remove $(1,859)=9.20, p<.005, \eta^{2}=.01$, with $95 \% \mathrm{CI}$ from 0 to .03 .

Gender Differences. We did not expect strong gender differences. However, we hypothesized that if such differences did exist, they would be manifest on three factors on which the groups of 375 women would be expected to place higher importance than the group of 493 men: primacy of rights of human participants, IRB outreach, and interactional justice. No significant multivariate gender differences were found, $p=.37, \eta^{2}=0$.

\section{Relationships Among Groups}

We applied binary logistic regression to test the hypotheses that rating of an investigator's own IRB (relative to the ideal) would score higher for biomedical than social/ behavioral investigators, and that the relationship would 

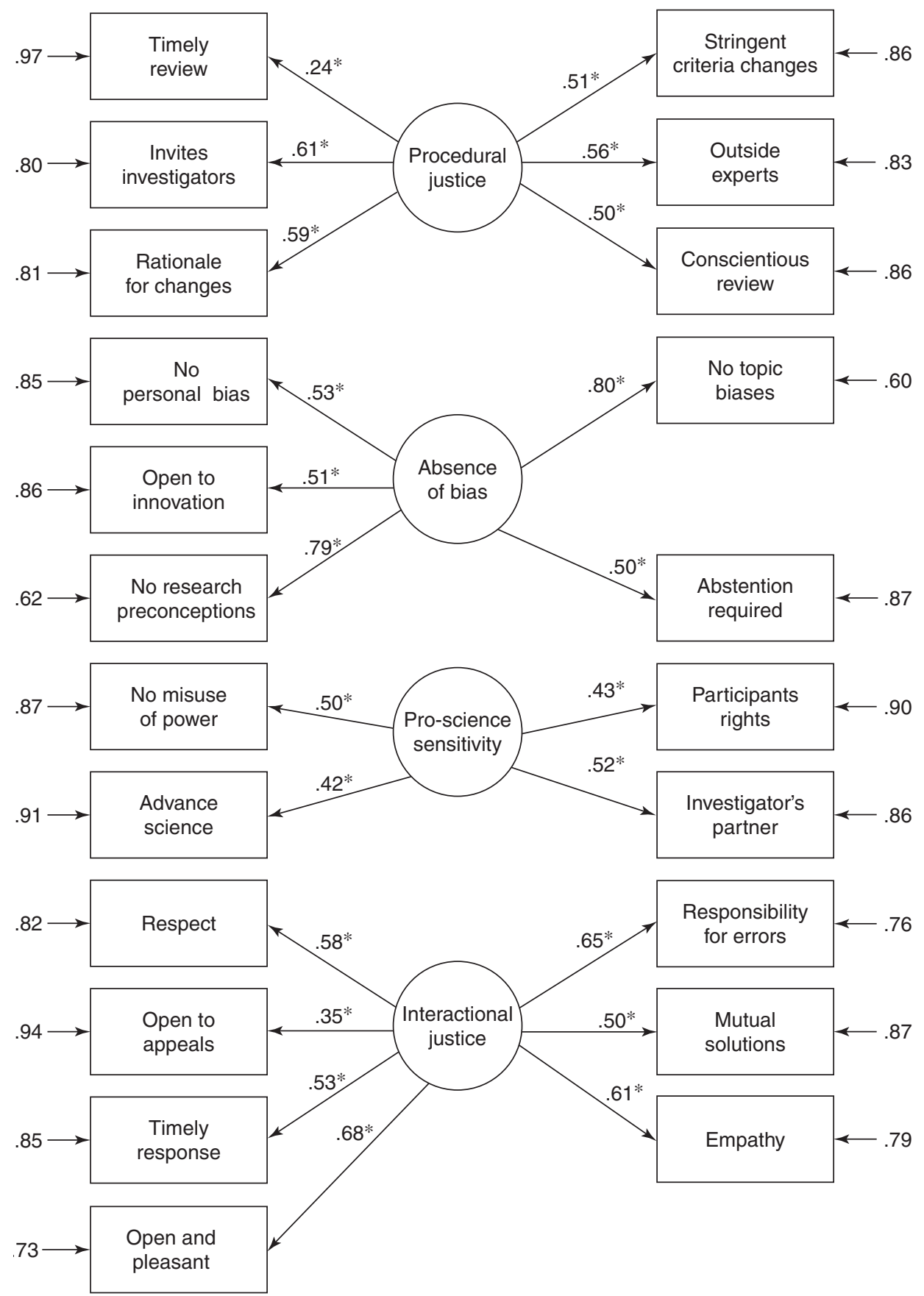

${ }^{*} P<.05$

FIG. 1. Eight-factor model of importance of IRB characteristics, RMSA = .059. Factor correlations are in Table 2.

rate more strongly within the university setting than other settings. Rating of the investigator's own IRB was dichotomized by combining the three negative (very much unlike..., unlike..., and somewhat unlike an ideal IRB) vs. the three positive categories (very much similar..., similar..., and somewhat similar to an ideal IRB). Four-year colleges were omitted and remaining categories combined to create a dichotomy between 

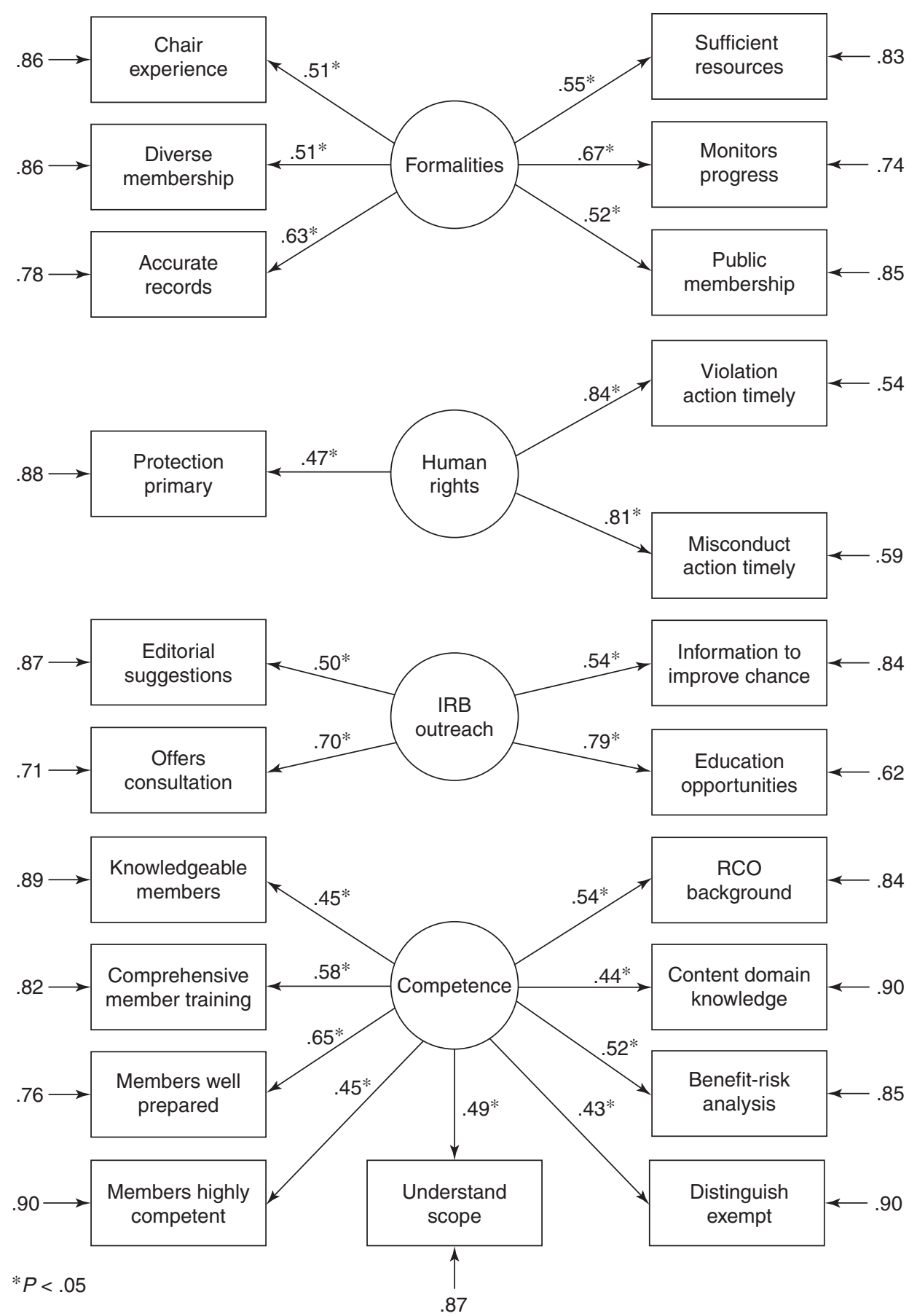

FIG. 1. (Continued)

universities and other settings. Data were available from 704 investigators for this analysis. Neither the main effects of university setting nor investigator type nor the interaction between setting and investigator type successfully predicted rating of the investigator's own IRB, $p>.05, R^{2}=.01$.

The hypothesis that investigators who do more exempt research (tested using both breakdowns previously 
TABLE 2. Correlations among factors.

\begin{tabular}{|c|c|c|c|c|c|c|c|}
\hline Factor & $\begin{array}{l}\text { Absence } \\
\text { of Bias }\end{array}$ & $\begin{array}{l}\text { Pro-science } \\
\text { Sensitivity }\end{array}$ & $\begin{array}{l}\text { Interactional } \\
\text { Justice }\end{array}$ & Formalities & $\begin{array}{l}\text { Human } \\
\text { Rights }\end{array}$ & $\begin{array}{c}\text { IRB } \\
\text { Outreach }\end{array}$ & Competence \\
\hline Procedural justice & $.723 *$ & $.708 *$ & $.776 *$ & $.684^{*}$ & $.529 *$ & $.651^{*}$ & $.892 *$ \\
\hline Bias & & $.714^{*}$ & $.641 *$ & $.529 *$ & $.342 *$ & $.442 *$ & $.670 *$ \\
\hline Pro-science sensitivity & & & $.821 *$ & $.393^{*}$ & $.183^{*}$ & $.463^{*}$ & $.649 *$ \\
\hline Interactional justice & & & & $.646 *$ & $.322 *$ & $.683^{*}$ & $.749 *$ \\
\hline Formalities & & & & & $.735^{*}$ & $.720 *$ & $.937^{*}$ \\
\hline Human rights & & & & & & $.395^{*}$ & $.639 *$ \\
\hline IRB outreach & & & & & & & $.697^{*}$ \\
\hline
\end{tabular}

${ }^{*} p<.001$

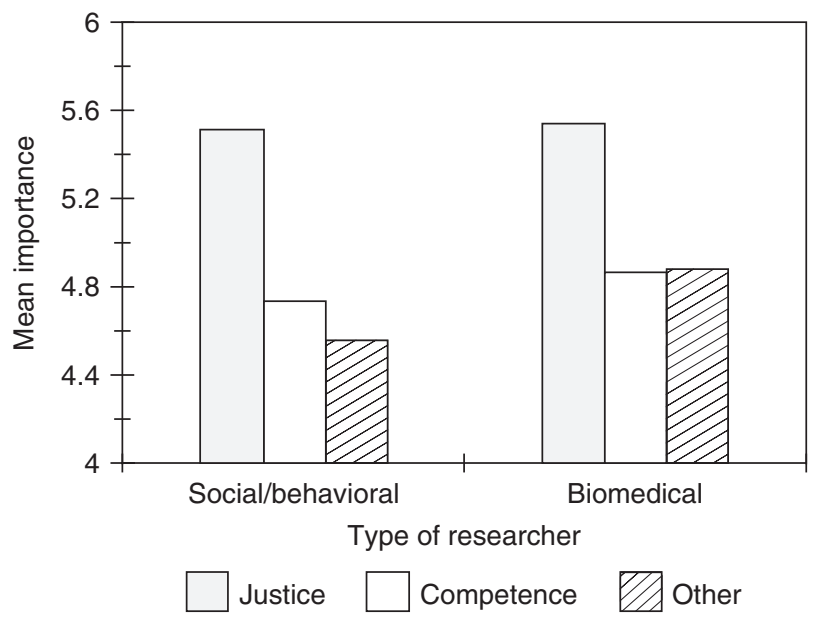

FIG. 2. Average importance of justice, competence, and other issues for social/behavioral and Biomedical Researchers

described in separate $2 \times 2 \chi^{2}$ analyses) would rate their own IRB lower than those who do less exempt research also was not borne out, $p>.05$.

\section{Discussion and Summary}

\section{Are Fairness and Consideration of IRBs Important to Investigators?}

Our primary interest was to learn if organizational justice issues rate as critically important to investigators as they do for people in more traditional employment settings, despite substantial differences between the two work venues. Most of the items on the survey were rated as at least somewhat important to both biomedical and social behavioral investigators, with biomedical investigators finding the items slightly more important. However, the four justice factors (procedural justice, interactional justice, lack of bias, and pro-science
TABLE 3. Comparisons among justice factors: mean differences/ partial $\eta^{2}$. (95\% confidence interval around partial $\eta^{2}$ in parentheses.)

\begin{tabular}{lccc}
\hline Factor & $\begin{array}{c}\text { Absence } \\
\text { of Bias }\end{array}$ & $\begin{array}{c}\text { Pro-science } \\
\text { Sensitivity }\end{array}$ & $\begin{array}{c}\text { Interactional } \\
\text { Justice }\end{array}$ \\
\hline Procedural & $0.30 * / .18$ & $0.12 * / .02$ & $<.01 / .00$ \\
justice & $(.14-.23)$ & $(.01-.04)$ & $(0.0-.00)$ \\
Absence & & $-0.27 * / .08$ & $-0.39 * / .17$ \\
of Bias & & $(.04-.14)$ & $(.09-.29)$ \\
Pro-science & & & $-0.12 * / .02$ \\
sensitivity & & & $(.01-.03)$ \\
${ }^{*} p<.001$ & & &
\end{tabular}

sensitivity) scored as more important than other IRB characteristics, with procedural and interactional justice rated as even more significant than the absence of bias and pro-science sensitivity. It may come as a surprise that timeliness, open communication, conscientiousness, and interpersonal sensitivity are of paramount importance to investigators, whereas many of the ways IRBs may define themselves are rated as less important. For example, upholding the rights of participants as the sole function of IRBs, outreach offerings (e.g., seminars on how to apply for grant funding) and how IRBs are structured, while not viewed as unimportant, seem to hold less importance to investigators than feeling that they have been fairly and respectfully treated.

One of the few statistical differences found to differentiate social behavioral researchers from biomedical researchers suggests that social behavioral researchers rated justice issues as more important. We did not predict this finding, but it could be that social behavioral researchers are more attuned to interpersonal issues, matters of equity, and organizational performance because these subject matters are part of their study domain. Also, the medical research environment is generally more hierarchical and accepting of authority than the social behavioral research environment, and 
social and behavioral researchers often complain that they are held to standards reasonably applicable to biomedical research, but unduly strict when applied to them (Cohen, 1998).

We felt heartened to learn that the majority of the respondents felt positively about their IRBs. Only $10.4 \%$ reported decidedly negative views about their own IRBs. When we compared investigators who expressed some level of dissatisfaction with their IRBs to those who felt generally satisfied, however, the sole difference focused on the importance of interactional justice. This finding holds particular interest because it suggests that a primary reason for investigators' dissatisfaction with their IRBs occurs because they perceive themselves as being treated disrespectfully. It may behoove IRBs to remain especially sensitive to the feelings of investigators when communicating with them, particularly if extensive changes are required or if a protocol is not approved. As organizational research has repeatedly reported, even criticism and disappointing news can be experienced as acceptable if the recipient feels treated with dignity and is given a full explanation of the reasons for a negative decision.

\section{Where No Differences Were Found}

Many of the demographic differences we predicted did not materialize. We hypothesized that those accustomed to full protocol reviews may also have more patience and a greater tolerance for scrutiny, whereas those whose work falls into the exempt category may complain when swift approval of their work does not occur. However, no differences emerged. The frequently stated complaints by social behavioral investigators that their low risk research is often held to the same standards as higher risk biomedical research prompted our hypothesis that biomedical investigators would rate their own IRBs more favorably than would social and behavioral science investigators, but this did not occur.

Because IRBs at research universities may be better organized and staffed, we hypothesized that investigators at research universities would rate their own IRBs higher than would investigators from other settings. This hypothesis was not borne out. We assumed that those who had not recently served on IRBs would be more concerned with justice issues and rate them higher than would those who have recently served. Our original thinking was that upset investigators might be annoyed rather than sympathetic to those serving on IRBs. However, this hypothesis was not borne out either. We also expected that those who had served on IRBs within the last 5 years would give higher rating to ensuring the rights of human participants and the formalities associated with running an IRB, but there were no differences. We assumed that those investigators who had fewer years of research experience would have somewhat different needs from their IRBs, but no differences emerged. We did not predict sex differences, and there were none. In sum, it appears that the differences in importance of IRBs characteristics override generalizations that might be made about characteristics of researchers.

\section{Practical Implications}

Strong associations exist between the perception of fair and just employers' policies and employees' good citizenship behaviors (Folger \& Cropanzano, 1998). Conversely, when management is perceived to be unfair or unjust, employees often have negative reactions, including the commission of dishonest acts (Folger, 1993; Robinson \& Bennett, 1995; Miller, 2001; Youngblood, Trevino, \& Favia, 1992). When a decisionmaking process becomes perceived as unjust, the level of employee commitment goes down, the amount of theft or other forms of retaliation rises, and more people look for work elsewhere (Cropanzano \& Greenberg, 1997; Greenberg \& Scott, 1996; Skarlicki \& Folger, 1997). Assuming that these dynamics can be generalized, satisfied investigators are important to both IRBs as well as institutions they represent.

What forms of negative reactions might occur when investigators perceive their IRBs as unjust? Dishonest behavior is one such possibility. Scientific misconduct is a serious matter, and conventional wisdom attributes its causes to investigators' excessive ambition and poor character. Paradoxically, however, an IRB perceived as unfair, incompetent, arrogant or biased, could impel an investigator to break rules to ensure ethical science. For example, one investigator experienced numerous problematic interactions with his IRB. Most involved what he regarded as persnickety demands for alterations that compromised his designs. Now he includes elaborate, detailed content he knows will bore readers while omitting or distorting elements of his protocol that he believes might cause his IRB to recoil. He reports that this tactic has consistently worked (Keith-Spiegel \& Koocher, 2005).

Investigators have invested much of themselves in their work, so having their work judged as unsatisfactory is likely experienced as a personal affront. According to Folger and Cropanzano (1998), if the insult is sufficiently strong, people will act against their own self-interest and become heavily emotionally invested in getting back at those who offended them 
(Skarlicki \& Folger, 1997). Furthermore, organizational research confirms that acting out in dishonest ways often feels justifiable to those who feel victimized or dismissed by a source of authority, especially if they compare themselves to others who have apparently received better treatment (Folger, 1984). Angry, insulted investigators may be more likely to behave in ways that are detrimental to the IRB, the institution, and their scientific work. For example, investigators dissatisfied with their IRBs could submit incomplete protocols, omitting content or procedures they believe might lead to disapproval, saying one thing and actually doing another, or not submitting their protocols at all (KeithSpiegel \& Koocher, 2005). Conceivably risks to participants could go forward unchecked.

Finally, our response rate of 39\% leaves open the possibility of a non-response bias.

\section{Best Practices}

The ideal ethics committee appears to be a just body that employs fair procedures, treats investigators with respect, and accords them the opportunity to have a voice when disagreements arise. This finding overwhelms the few mostly small differences between social/behavioral scientists and biomedical scientists. Our work strongly suggests that proactive measures could enhance the perception of an ethics committee as fair and respectful.

Materials and programs for training committee staff and members should focus on ways to ensure that investigators feel appreciated and respected, and do not feel demeaned, shut out, or mistreated. Skills of interacting with upset or angry investigators and organizational policies and procedures that promote the perception of just treatment should be developed. Organizational image management practices should be considered and adapted so that genuine concerns for maintaining fair relationships with investigators are clearly perceived by investigators.

The implementation of measures to enhance the perception of fairness may improve an institution's overall research program. Universities, research hospitals, and other research institutions vigorously promote scientific productivity. Yet, continuing problems with scientific dishonesty have created the need for stronger controls and monitoring that could inadvertently stunt research. We believe that a climate that communicates justice and fairness to investigators will help deter unethical behavior. Similarly, Swazey (1978) observed that external controls are surely necessary, but she also recognized inherent limitations.

\section{Research Agenda}

The major finding of this paper is that investigators' perception of the fairness of their ethics committee is central to their evaluation of its performance. This, coupled with comments by some of our respondents and the findings of others, strongly suggests that the perception of unfairness will motivate some investigators to engage in subterfuge and misconduct designed to "level the playing field." This implication underlines the need for research on most effective ways to improve the committee's image in the eyes of the investigators it serves. Such experimental interventions require discovery of what needs to be improved, some approaches to creating those improvements, and post-experimental assessment of how the intervention affected investigators' perception of the committee. Fortunately, the research reported here provides the measurement tools that such an experiment would require. The assessment tools and a user guide may be accessed at www.ethicsresearch. com. The authors welcome feedback on the use of the IRB Researcher Assessment Tool (or IRB RAT for short).

IRBs can adapt the RAT to assess their investigators' evaluation of which aspects of the committee's performance they consider important and which, among those aspects, they perceive as needing improvement. This diagnostic information, in turn, can be used to guide the selection and development of an experimental intervention designed to provide the needed improvement. Following implementation of that intervention, the survey can again be administered, and the comparison of pre- and post-test data would provide both an overall evaluation of the success of the interventionan indication of the areas that benefited most from the intervention and those that remain in need of further work. A second useful comparison would be with the findings reported herein, allowing institutions to learn how their investigators' perceptions compare with the results of a larger external sample. A third useful comparison would be to obtain responses to the survey from ethics committee managers, staff and other members who are part of the human research protection program and not otherwise involved in research. How do those who are focused on compliance differ from investigators in their perception of the committee? How do they regard the investigators? These differences may provide useful insight into kinds of changes in committee perceptions, policies, and interpersonal skills that are in need of modification.

Individual ethics committees may differ from one another in their strengths and weaknesses and in the 
ways their investigators perceive them. Nevertheless, the descriptions of experimental interventions that have been implemented, and evaluations of the extent to which they succeeded would be extremely valuable to other ethics committees elsewhere. The development of such a literature upon which each succeeding intervention could build would yield an ever more valuable body of knowledge about the organizational behavior and perceived fairness of ethics committees.

Once more has been learned about whether or how reputation for fairness can be improved, the next step would be to investigate whether there is indeed a causal link between the perception of unfairness and willingness to engage in minor misconduct intended to level the playing field. That is, can changes in the ethics committee's fairness and in the perception of its fairness improve the willingness of investigators to play by the rules of the committee? A variety of indirect queries (e.g., what do you think other people do?) and direct, but clearly anonymous queries (e.g., how would you handle a situation in which the ethics committee objected to some aspect of your protocol?) would be expected to reflect actual behaviors or tendencies. Such research could be directed to new faculty who are active researchers and have an extensive recent history of interacting with the committee, rather than senior faculty whose attitudes may reflect long-held beliefs and experience. Such an assessment of investigator behavior or attitude would obviously need to be conducted by investigators whose work is clearly independent of the ethics committee. Two types of design might be used to learn whether attitudes change quickly once they are in place: one in which the same, more senior investigators take the pre- and post-test; the other in which brand new investigators take the post-test only and their performance is compared with the pre- and post-test performance of their slightly more senior peers.

A second major extension of this line of research would be to learn how investigators respond to rejection by journal editors and funders of research. Is the importance of organizational justice as relevant to the perception of journal editors and funding foundation review panels as it appears to be for ethics committees? When work is accepted, recipients probably do not think much about the decision processes involved. But, because grants and publications are of critical importance to most researchers, rejections are most unwelcome. How do such rejections affect investigators' perceptions of the decision processes that were involved, and how do investigators respond? Is there a similar "leveling of the playing field?" What can be done by these external institutions to change perceptions of unfairness?

\section{Implications for Education}

What are effective ways to introduce ethics committees to the concepts developed here? Ideally, any education of the IRB in concepts of justice would be undertaken in collaboration with faculty who specialize in organizational development or organizational justice, and who have in depth understanding of the operation of the IRB system.

One approach would be to educate committee members and staff in the principles of procedural and interpersonal justice, and to conduct workshops in which the committee develops policies, procedures and training designed to improve on these aspects of justice.

A second approach would involve the committee members and staff in a self-evaluation project using the survey, perhaps in modified form, to evaluate how the IRB perceives itself compared with how it is perceived by its clientele; plans could then be developed for responding to the findings.

A most effective educational project would be to combine these two approaches, and ultimately to create and carry out whatever intervention seemed to be required, and to conduct the pre- posttest design experiment described in the research agenda above.

\section{Acknowledgments}

This article is based on a project titled The Relationship Between Perceived Organizational Justice and Scientific Dishonesty, funded by a cooperative grant from the Office of Research Integrity and NIH, Grant No. R01 NS42454, Children's Hospital, Boston.

\section{Author Note}

Address correspondence to: Patricia Keith-Spiegel, 3386 Haas Drive, Aptos, CA 95003. E-MAIL: pksipegel@ comcast.net

\section{Author Biographical Sketches}

Patricia Keith-Spiegel the Voran Honors Distinguished Professor of Social and Behavioral Sciences, Emerita, at Ball State University and an Consulting Editor of Ethics $\&$ Behavior. She is a past chair of the Ethics Committee of the American Psychological Association and has written extensively in the areas of academic dishonesty 
and research and professional ethics, including Ethics in Psychology with Gerald Koocher. She has served as President of the Western Psychological Association and the Society for the Teaching of Psychology, Division 2 of the American Psychological Association.

Gerald P. Koocher is Dean of the School for Health Studies and Professor of Psychology at Simmons College, with teaching appointments at Boston College and Harvard Medical School. He is president of the American Psychological Association in 2006, and holds five specialty diplomas from the American Boards of Professional Psychology (Clinical, Clinical Child/ Adolescent, Family, Forensic, and Health Psychology). Koocher founded and edits the journal Ethics \& Behavior and previously edited the Journal of Pediatric Psychology and The Clinical Psychologist. Books he has authored include the Psychologist's Desk Reference.

Barbara Tabachnick is Professor Emerita at California State University, Northridge. She is co-author of two major textbooks: Using Multivariate Statistics (5th Ed.) and Experimental Designs Using ANOVA. Over the last 40 years, Dr. Tabachnick has provided consultation in research design and statistical data analysis on numerous diverse research projects in fields such as professional ethics, transportation, and biomedical research. She provides workshops in application of statistical software to advanced data analysis for a variety of organizations.

\section{End Notes}

${ }^{1}$ Permission was obtained from the manager of the DHHS Computer Retrieval of Information on Scientific Projects (CRISP) data base and the APS staff to approach randomly selected members on their lists. ${ }^{2} \mathrm{EQS}$ Version 6, a structural equation modeling program, was used to impute missing values, which were scattered over items and participants (a few participants failed to complete the second page of the questionnaire). No item was missing more than $2.2 \%$ of its values. Severe departure from normality for most of the items was dealt with by using robust statistics in estimating and testing parameter estimates for factor models. A test of the correlation matrix showed it to be highly factorable with Bartlett's sphericity $\chi 5(990, N=886)=14396.3, \mathrm{p}<.001$ and Kaiser-Meyer-Olkin measure of sampling adequacy equaling .915 .

${ }^{3}$ Numbers preceding each item indicate its overall importance ranking.

\section{References}

BIES, R. J. (1987). Beyond "voice": The influence of decisionmaker justification an sincerity of procedural fairness judgments. Representative Research in Social Psychology, 17, 3-17.

BIES, R. J., Shapiro, D L., \& Cummings, L. L. (1988). Causal accounts and managing organizational conflicts: Is it enough to say it's not my fault? Communications Research, 15, 381-399.

CoHEN, J. (Nov., 1998). The federal perspective on IRBs. APS Observer, 5, 19.

Cropanzano, R. \& Greenberg, J. (1997). Progress in organizational justice: Tunneling through the maze. In C. L. Cooper \& I. T. Robertson (Eds.), International review of industrial and organizational psychology (pp. 317-372). New York: Wiley.

DuBois, J. M. \& JAmEs, M. (2004). Is compliance a professional virtue of researchers? Reflections on promoting the responsible conduct of research. Ethics \& Behavior, 14, 383-395.

Edwards, S. J. L., Kirchin, S., \& Huxtable, R. (2004). Research ethics committees and paternalism. Journal of Medical Ethics, 30, 88-91.

Ferraro, F. R., Szigeti, E., Dawes, K. J., \& Pan, S. (1999). A survey regarding the University of North Dakota Institutional Review Board: Data, attitudes, and perceptions. Journal of Psychology, 133, 272-276.
Folger, R. (1984). Perceived injustice, referent cognitions, and the concept of comparison level. Representative Research in Social Psychology, 14, 88-108.

FolgeR, R. (1993). Reactions to mistreatment at work. In K. Murnighan (Ed.), Social psychology in organizations: Advances in theory and research (pp. 161-183). Englewood Cliffs, NJ: Prentice Hall.

Folger, R. \& Cropanzano, R. (1998). Organizational justice and human resource management. Thousand Oaks, CA: Sage Publications.

Gray, B. H., Cooke, R. A., \& Tannenbaum, A. S. (1978). Research involving humans subjects. Science. 201, 1094-1101.

GREENBERG, J. (1993). Stealing in the name of justice. Organizational Behavior and Human Decision Processes, 54, 81-103.

Greenberg, J. (1996). The quest for justice on the job. Thousand Oaks, CA: Sage Publications.

GreENBERG, J. \& SCOTT, K. S. (1996). Why do workers bite the hands that feed them? Employee theft as social a exchange process. In B. M. Staw \& L. L. Cummings (Eds). Research in organizational behavior (Vol. 18 pp. 111-166). Greenwich, CT: JAI Press.

Hayes, G. J., Hayes, S. C., \& Dykstra, T. (1995). A survey of university institutional review boards: Characteristics, policies, and procedures. IRB: A Review of Human Subjects Research, 17, 1-6. 
Kancelbaum, B. (2002). Social scientists and institutional review boards. Social Science Research Council, 3, 1-5.

Keith-Spiegel, P. \& Koocher, G. P. (2005). The IRB paradox: Could the protectors also encourage deceit? Ethics \& Behavior, 15(4), 339-349.

Leventhal, G. S. (1976). The distribution of rewards and resources in groups and organizations. In L. Berkowitz \& W. Walster (Eds.), Advances in experimental social psychology (Vol. 9, pp. 91-131). New York: Academic Press.

Leventhal, G. S. (1980). What should be done with equity theory? In K. J. Gergen, M. S. Greenberg \& R. H. Willis (Eds.), Social exchanges: Advances in theory and research (pp. 27-55). New York: Plenum.

Lind, E. A., \& Tyler, T. R. (1988). The social psychology of procedural justice. New York: Plenum.

Malouff, J. M., \& Schutte, N. S. (2005). Academic psychologists' perspective on the human research ethics review process. Australian Psychologist, 40, 57-62.

Miller, D. T. (2001). Disrespect and the experience of injustice. Annual Review of Psychology, 527-553.

OHRP/OPPR. (2000a) OHRP Compliance Activities: Common Findings and Guidance. Washington D.C., Author. Retrieved October 3, 2000 from the World Wide Web: ohrp.osophs. dhhs.gov/polasur.htm

OHRP/OPPR. (2000b) Compliance oversight procedures. Washington D.C., Author. Retrieved August 30, 2000 from the World Wide Web: http://ohrp.osophs.dhhs. gov/polasur.htm PALCA, J.(1996). Institutional review boards: A net too thin. Hastings Center Report, 26, 4.

Robinson, S. L., \& BENNETT, R. J. (1995). A typology of deviant workplace behaviors: A multidimensional scaling study. Academy of Management Journal, 38, 555-572.
Silberner, J. (1998). Remodelling IRBs. Hastings Center Report, $28,5$.

SkARLICKI, D. P. \& Folger, R. (1997). Retaliation in the workplace: The roles of distributive, procedural, and interactional justice. Journal of Applied Psychology, 82, 434-443.

SWAZEY, J. P. (1978). Protecting the animal of necessity: Limits to inquiry in clinical investigations. Daedalus, 107, 129-145.

Thibaut, J. W. \& Walker, L. (1975). Procedural justice: A psychological perspective. Hillsdale, NJ: Lawrence Erlbaum.

TYLER, T. R., \& BIES, R. J. (1989). Beyond formal procedures: The interpersonal context of procedural justice. In J. S. Carroll (Ed.). Applied social psychology and organizational settings (pp. 77-98). Hillsdale, NJ: Erlbaum.

Tyler, T. R., \& Lind, E. A. (1992). A relational model of authority in groups. In M. P. Zanna (Ed.). Advances in experimental social psychology (Vol. 25, pp. 115-191). San Diego, CA: Academic Press.

Tyler, T. R., RAsinSKI, K., \& SPODICK, N. (1985). Influence of voice and satisfaction with leaders: Exploring the meaning of process control. Journal of Personality and Social Psychology, $42,333-344$

WAINWRIGHT, P. \& SAUNDERS, J. (2004). What are local Issues? The problem of the local review in research. Journal of Medical Ethics, 30, 313-317.

WeINER, B. (1995). Judgments of responsibility. New York: Guilford Press.

Youngblood, S. A., Trevino, L. K., \& FAVIA, M. (1992). Reactions to unjust dismissal and third-party dispute resolution: A justice framework. Employee Responsibility and Rights Journal, 5, 283-307. 
